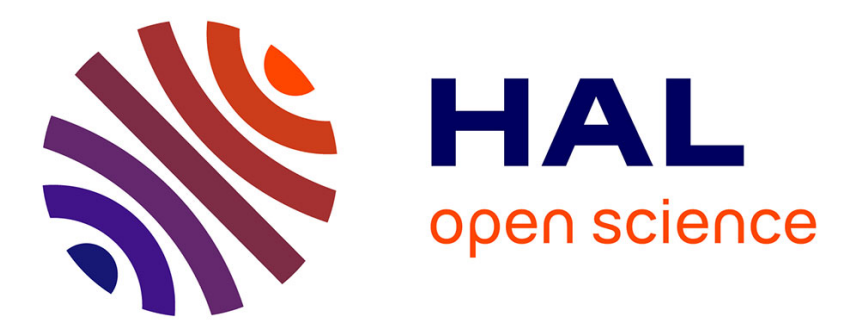

\title{
Hermite MLS Approximation for Discretizing Fourth Order Partial Differential Equations Encountered in Beam and Plate Models
}

P. Joyot, Francisco Chinesta, Pierre Villon, Daniel Batal Khoshnoudirad

\section{- To cite this version:}

P. Joyot, Francisco Chinesta, Pierre Villon, Daniel Batal Khoshnoudirad. Hermite MLS Approximation for Discretizing Fourth Order Partial Differential Equations Encountered in Beam and Plate Models. 10th ESAFORM Conference on Material Forming, 2007, Saragosse, Spain. pp.1384-1389, 10.1063/1.2729708 . hal-01008249

\section{HAL Id: hal-01008249 \\ https://hal.science/hal-01008249}

Submitted on 14 Feb 2018

HAL is a multi-disciplinary open access archive for the deposit and dissemination of scientific research documents, whether they are published or not. The documents may come from teaching and research institutions in France or abroad, or from public or private research centers.
L'archive ouverte pluridisciplinaire HAL, est destinée au dépôt et à la diffusion de documents scientifiques de niveau recherche, publiés ou non, émanant des établissements d'enseignement et de recherche français ou étrangers, des laboratoires publics ou privés.

\section{다)(1) $(5$}

Distributed under a Creative Commons Attribution - NonCommerciall 4.0 International 


\title{
Hermite MLS approximation for discretizing fourth order partial differential equations encountered in beam and plate models
}

\author{
P. Joyot*, F. Chinesta ${ }^{\dagger}$, P. Villon** and B. Khoshnoudirad ${ }^{* \dagger}$ \\ ${ }^{*}$ LIPSI, ESTIA technopole izarbel, F-64210 Bidart, France \\ ${ }^{\dagger}$ LMSP UMR 8106 CNRS-ENSAM, 151 Boulevard de l'Hopital, F-75013 Paris, France \\ ${ }^{* *}$ UTC, BP 20529, F-60205 Compiégne Cedex, France
}

\begin{abstract}
In this work we propose a novel strategy for discretizing beam and plate models in the framework of a meshless Hermite moving least square approach which allows a natural and simple enforcement of boundary conditions.
\end{abstract}

Keywords: meshless method, moving least square, hermite moving least square, beam PACS: $02.60 . \mathrm{Cb}, 82.20 . \mathrm{Wt}$

\section{INTRODUCTION}

In this work we focus on the numerical treatment of structural problems involving plate and beam models using standard and enriched moving least squares approximations.

Several researchers have studied the use of meshfree solution techniques for beam and plate problems (see [1] for references). In [2], the element free Galerkin (EFG) method was used and Lagrange multipliers were used to apply the essential boundary conditions. In [3], the meshless local Petrov-Galerkin (MLPG) technique was used for the solution of $1 \mathrm{D}$ beam problems using the generalized moving least squares (GMLS) interpolation.

In this work we considered a Hermite moving least square approximation (HMLS) associated with a Galerkin discretization. Thus, different degrees of freedom are associated to each node consisting of the unknown field and some of its derivatives.

The definition of the HMLS and GMLS approximations are very close. The main difference lies in the polynomial basis scaling that allows for optimal rates.

This procedure allows a simple enforcement of the boundary conditions, in particular those related to the rotations, avoiding the necessity of proceeding by introducing these boundary conditions via the use of Lagrange multipliers, as usually performed in the standard moving least square technique (see [2]).

In the first part of this work we introduce the Hermite moving least squares formulation, and then the problematic related to the boundary conditions enforcement is addressed. Finally some examples allows to appreciate the potentialities of the proposed strategy. 


\section{HERMITE MLS APPROXIMATION}

For the sake of simplicity from now, the strategy will be described in the onedimensional case.

Let $\chi=\left\{\begin{array}{lll}x_{1} & \cdots & x_{n}\end{array}\right\}$ be a cloud of $n$ nodes. Function $u^{e x}(x)$ is defined from its nodal values. Thus, at each node $x_{i}$, we consider two degrees of freedom $\boldsymbol{u}_{i}^{T}=$ $\left[\begin{array}{cc}u_{i}^{u} & u_{i}^{x}\end{array}\right]$. The first one concerns the field $u^{e x}(x)$ and the second one its derivative. We define the unknown vectors containing the nodal degrees of freedom as $\mathscr{U}^{T}=$ $\left[\begin{array}{llllll}u_{1}^{u} & \cdots & u_{n}^{u} & u_{1}^{x} & \cdots & u_{n}^{x}\end{array}\right]$.

The aim of the approximation is the representation of $u^{e x}(x)$ from $\mathscr{U}$. We denote by $u^{a p p T}(x)=\left[\begin{array}{ll}u^{u a p p}(x) & u^{x a p p}(x)\end{array}\right]$ the degrees of freedom related to the reconstructed function.

Let $x$ be an evaluation point and $y$ another point located in the neighborhood of $x$. We define the local approximation $v_{u}(x, y)$ centered at $x$ and evaluated at $y$ by

$$
v_{u}(x, y)=P^{u T}\left(\frac{y-x}{a}\right) \boldsymbol{\theta}(x)
$$

where $P^{u T}(x)=\left[\begin{array}{llll}1 & x & \cdots & \frac{1}{p^{!}}(x)^{p}\end{array}\right]$ is a polynomial basis of degree $p$, and $a$ a scale factor.

The diffuse derivative of the approximation can be written as

$$
\frac{d}{d y} v_{u}(x, y)=a^{-1} P^{x T}\left(\frac{y-x}{a}\right) \boldsymbol{\theta}(x)
$$

with $P^{x T}(x)=\left[\begin{array}{llll}0 & 1 & \cdots & \frac{1}{(p-1) !}(x)^{p-1}\end{array}\right]$. Writing $v_{x}(x, y)=a \frac{d}{d y} v_{u}(x, y)$, equations (1) and (2) lead to

$$
\left[\begin{array}{c}
v_{u}(x, y) \\
v_{x}(x, y)
\end{array}\right]=P^{T}\left(\frac{y-x}{a}\right)\left[\begin{array}{c}
\theta_{0}(x) \\
\vdots \\
\theta_{p}(x)
\end{array}\right]
$$

with $P(x)=\left[\begin{array}{ll}P^{u}(x) & P^{x}(x)\end{array}\right]$. Thus, the second degree of freedom represents the diffuse derivative multiplied by the scale factor $a$.

The reconstructed function $\boldsymbol{u}^{\text {app }}(x)$ is defined by

$$
\begin{aligned}
\boldsymbol{u}^{a p p}(x) & =\boldsymbol{v}(x, x) \\
& =P^{T}(0) \boldsymbol{\theta}(x)
\end{aligned}
$$

Vector $\boldsymbol{\theta}$ minimizes

$$
\begin{array}{r}
J(\boldsymbol{\theta}(x))=\frac{1}{2} \sum_{i \in I(x)}\left(v_{u}\left(x_{i}, x\right)-u_{i}^{u}\right)^{2} w_{r_{i}}\left(x_{i}, x\right) \\
+\frac{1}{2} \sum_{i \in I(x)}\left(v_{x}\left(x_{i}, x\right)-u_{i}^{x}\right)^{2} w_{r_{i}}\left(x_{i}, x\right)
\end{array}
$$


where $w_{r_{i}}\left(x_{i}, x\right)$ represents the weight function related to the node $x_{i}$, and $r_{i}$ is the radius of the domain of influence of $w_{r_{i}}\left(x_{i}, x\right)$. Equation (5) can be also written as

$$
J(\boldsymbol{\theta}(x))=\frac{1}{2}(\mathscr{P}(x) \boldsymbol{\theta}(x)-\mathscr{U})^{T} \mathscr{W}(x)(\mathscr{P}(x) \boldsymbol{\theta}(x)-\mathscr{U})
$$

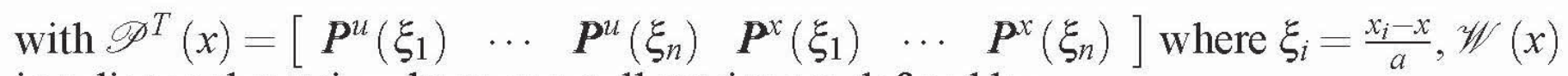
is a diagonal matrix whose non null entries are defined by

$$
\operatorname{diag}(\mathscr{W}(x))=\left[\begin{array}{llllll}
w_{r_{1}}\left(x_{1}, x\right) & \cdots & w_{r_{n}}\left(x_{n}, x\right) & w_{r_{1}}\left(x_{1}, x\right) & \cdots & w_{r_{n}}\left(x_{n}, x\right)
\end{array}\right]
$$

The value of $\boldsymbol{\theta}$ minimizing $J$ is derived by solving the system

$$
\boldsymbol{M}(x) \boldsymbol{\theta}(x)=\mathscr{P}^{T}(x) \mathscr{W}(x) \mathscr{U}
$$

with the moment matrix $M(x)$ defined by $M(x)=\mathscr{P}^{T}(x) \mathscr{W}(x) \mathscr{P}(x)$.

The approximation is defined by

$$
\begin{aligned}
\boldsymbol{u}^{a p p}(x) & =\sum_{i \in I(x)} N_{i}(x) \boldsymbol{u}_{i} \\
& =\mathscr{N}^{T}(x) \mathscr{U}
\end{aligned}
$$

where $N_{i}(x)$ define the matrix of the shape functions

$$
N_{i}(x)=\left[\begin{array}{ll}
N^{u u}{ }_{i}(x) & N^{u x}{ }_{i}(x) \\
N^{x u}{ }_{i}(x) & N^{x x}{ }_{i}(x)
\end{array}\right]
$$

and $\mathscr{N}^{T}(x)$ define the matrix containing all the shape functions

$$
\mathscr{N}^{T}(x)=\left[\begin{array}{cc}
\mathscr{N}^{u u T}(x) & \mathscr{N}^{u x T}(x) \\
\mathscr{N}^{x u T}(x) & \mathscr{N}^{x x T}(x)
\end{array}\right]
$$

with $\mathscr{N}^{\alpha \beta^{T}}(x)=\left[\begin{array}{lll}N^{\alpha \beta}{ }_{1}(x) & \cdots & N^{\alpha \beta}{ }_{n}(x)\end{array}\right]$.

The expression of $\mathscr{N}^{T}(x)$ is obtained from equations (4), (8), (9) by eliminating $\boldsymbol{\theta}(x)$. We obtain

$$
\mathscr{N}(x)=\mathscr{W}(x) \mathscr{P}(x) P(0)
$$

\section{DISCRETE FORM AND BOUNDARY CONDITIONS ENFORCEMENT}

Beam models can be written in the generic form

$$
\left\{\begin{array}{l}
\triangle^{2} u(x)=f(x) \quad x \in[0, L] \\
u(0)=u_{0}^{u} \\
u_{x}(0)=u_{0}^{x} \\
u(L)=u_{L}^{u} \\
u_{,}(L)=u_{L}^{x}
\end{array}\right.
$$


This model is solved by considering its associated variational formulation that is discretized by applying the just introduced Hermite moving least squares formulation, leading to the linear system:

$$
K \mathscr{U}=f
$$

In the context of a standard moving least square approximation it is well known that the enforcement of essential boundary conditions requires a particular treatment because the shape functions related to internal nodes do not vanish on the domain boundary.

In the context of the just developed Hermite MLS strategy there is another problem because the additional degree of freedom represents the diffuse derivative multiplied by the scale factor $a$.

To circumvent this difficulty we are transforming equation (14) in order to explicit the real derivatives.

For this purpose we define the matrix $\mathscr{A}$ which defines at each point the relation between the nodal degrees of freedom and the approximated values

$$
\mathscr{U}^{a p p}=\mathscr{A} \mathscr{U}
$$

or

$$
\left[\begin{array}{l}
\mathscr{U}^{u a p p} \\
\mathscr{U}^{x a p p}
\end{array}\right]=\left[\begin{array}{ll}
\mathscr{A}^{u u} & \mathscr{A}^{u x} \\
\mathscr{A}^{x u} & \mathscr{A}^{x x}
\end{array}\right]\left[\begin{array}{l}
\mathscr{U}^{u} \\
\mathscr{U}^{x}
\end{array}\right]
$$

with

$$
\mathscr{A}^{\alpha \beta}=\left[\begin{array}{ccc}
N^{\alpha \beta}{ }_{1}\left(x_{1}\right) & \cdots & N^{\alpha \beta}{ }_{n}\left(x_{1}\right) \\
\vdots & & \vdots \\
N^{\alpha \beta}{ }_{1}\left(x_{n}\right) & \cdots & N^{\alpha \beta}{ }_{n}\left(x_{n}\right)
\end{array}\right]
$$

We can also, from the derivative of $\mathscr{A}$, compute the real nodal derivatives from the nodal values

$$
\left[\begin{array}{l}
\mathscr{U}^{u a p p}{ }^{x} \\
\mathscr{U}^{x a p p}{ }_{, x}
\end{array}\right]=\left[\begin{array}{ll}
\mathscr{A}^{u u} & \mathscr{A}^{u x} \\
\mathscr{A}^{x u} & \mathscr{A}_{, x}^{x x}
\end{array}\right]\left[\begin{array}{l}
\mathscr{U}^{u} \\
\mathscr{U}^{x}
\end{array}\right]
$$

that are noted by

$$
\mathscr{U}^{a p p}{ }_{, x}=\mathscr{A}_{, x} \mathscr{U}
$$

We define now the matrix $\tilde{\mathscr{A}}$ as

$$
\left[\begin{array}{c}
\mathscr{U}^{u a p p} \\
\mathscr{U}^{u a p p}{ }_{, x}
\end{array}\right]=\left[\begin{array}{ll}
\mathscr{A}^{u u} & \mathscr{A}^{u x} \\
\mathscr{A}_{, x}^{u u} & \mathscr{A}_{, x}^{u x}
\end{array}\right]\left[\begin{array}{l}
\mathscr{U}^{u} \\
\mathscr{U}^{x}
\end{array}\right]
$$

that is also noted by

$$
\tilde{\mathscr{U}}^{a p p}=\tilde{\mathscr{A}} \mathscr{U}
$$

In this way, we have established a relation between the nodal degrees of freedom and the approximated values and their derivatives.

Now, the system (14) can be rewritten as

$$
\tilde{\mathscr{A}}^{-1^{T}} \boldsymbol{K} \tilde{\mathscr{A}}^{-1} \tilde{\mathscr{U}}^{a p p}=\tilde{\mathscr{A}}^{-1^{T}} \boldsymbol{f}
$$

making possible the direct enforcement of essential boundary conditions. 
We are analyzing the convergence of the proposed strategy. For this purpose we consider a beam subjected to the following boundary conditions: $u(0)=0, u_{x}(0)=0, u(L)=1$, $u_{, x}(L)=1$. Moreover, a uniform load is distributed along its length $f(x)=g$. The internodal distance will be noted by $h$.

The results depicted in figures 1 and 2 were obtained by considering $g=10, L=1$, $a=3.6 \times h, a_{i}=a$. The weight function was a cubic spline, $p=3$, and the numerical integration was performed by using 5 Gauss points in each subdomain, being 5 the number of subdomains between each two consecutive nodes.

Figure 1 represents the displacement field, whose associated error is depicted in figure 2 using different norms: $L^{\infty}, L^{2}$ and $H^{1}$.

We can notice that the convergence rates are in the norms $L^{\infty}, L^{2}$ and $H^{1}$ respectively $4.2,4.5,3.3$. These values correspond to the optimal ones.

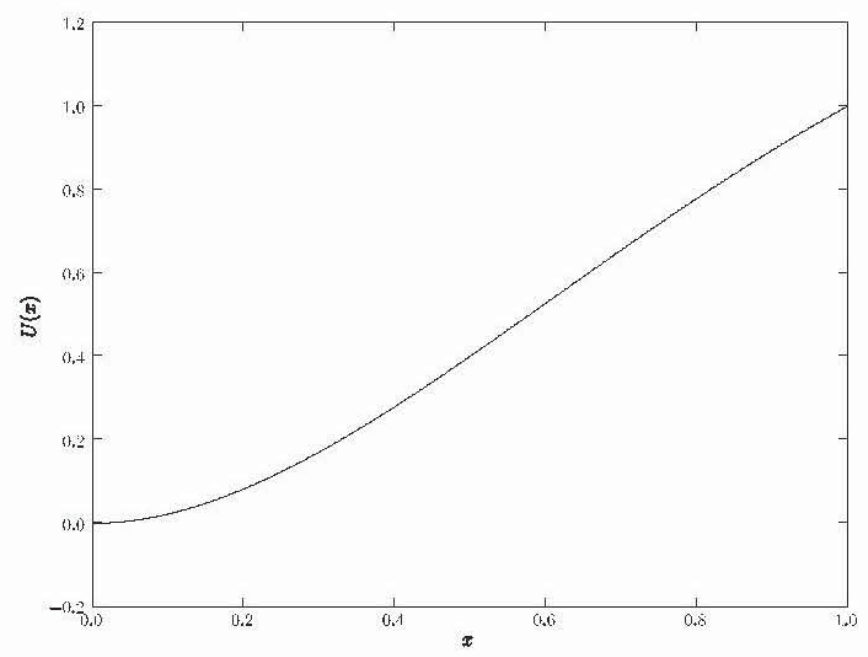

FIGURE 1. Displacement field $(n=15)$

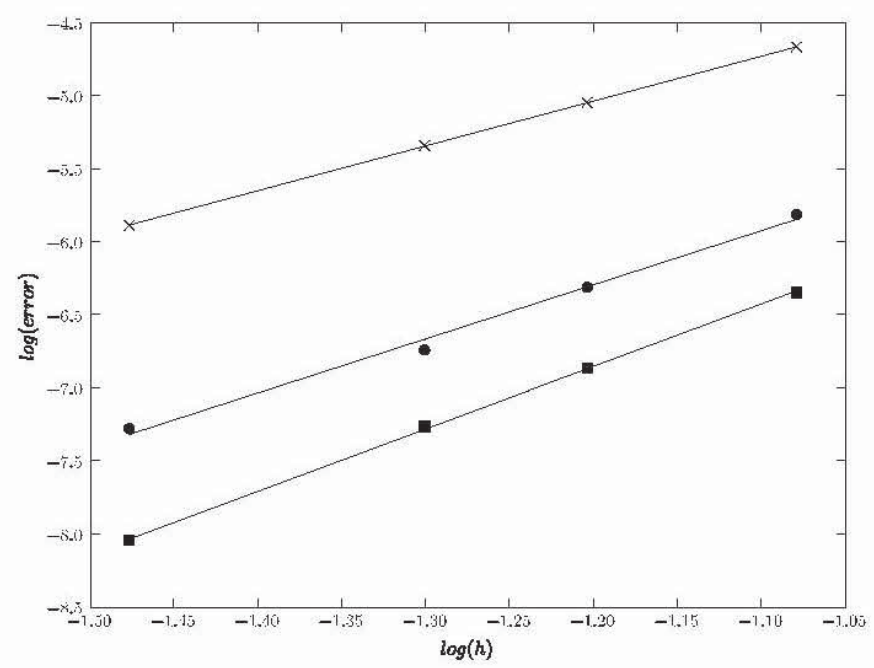

FIGURE 2. Convergence analysis, $L^{\infty}(\bullet), L^{2}(\boldsymbol{\square})$ and $H^{1}(\times)$ 


\section{CONCLUSIONS}

In this paper we have proposed a Hermite moving least squares discretization strategy specially adapted for solving models related to bi-harmonic differential operators as the ones encountered in models involving beam or plate structures. This approach allows to a simple, efficient and direct enforcement of boundary conditions. The preliminary convergence analysis shows optimal convergence rates.

In contrast to the classical and standard moving least squares approach, the Hermite variant increases the number of degrees of freedom by considering not only the nodal displacement, but also different field derivatives, with an unfavorable incidence in the computing time.

We are now developing a mixed approach that consists of a standard MLS approximation inside the domain but that becomes a Hermite-MLS when the domain boundary is approached. Thus, only extra-degrees of freedom are introduced at the nodes located in the boundary neighborhood allowing an accurate enforcement of boundary conditions. As the number of degrees of freedom is only slightly higher its impact on the CPU time will be no more significant.

\section{REFERENCES}

1. S. BaniHani and S. De. Development of a genetic algorithm-based lookup table approach for efficient numerical integration in the method of finite spheres with application to the solution of thin beam and plate problems. International Journal for Numerical Methods in Engineering, 67(12):1700-1729, 2006.

2. P. Krysl and T. Belytschko. Analysis of thin plates by the element-free galerkin method. Computational Mechanics, 17(1):26-35, 1995.

3. S. N. Atluri, J. Y. Cho, and H.-G. Kim. Analysis of thin beams, using the meshless local petrovgalerkin method, with generalized moving least squares interpolations. Computational Mechanics, 24(5):334-347, 1999. 